\title{
Caracterización molecular de Pseudomonas aeruginosa resistentes a carbapenémicos provenientes de cuatro hospitales de Venezuela
}

\author{
Armando Guevara, Carmen I. Sierra R. y Jacobus de Waard.
}

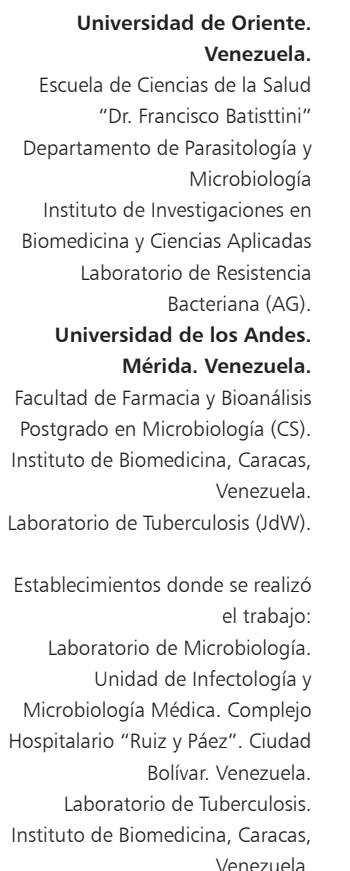

Conflictos de intereses: Ninguno Esta investigación fue parcialmente

financiada con recursos provenientes del proyecto $\mathrm{Cl}-5$ 040605-1622-09 del Consejo de Investigación de la Universidad de

Oriente, Venezuela y por el Proyecto LOCTI de Duty Free Americas C.A.

Recibido: 28 de marzo de 2012 Aceptado: 29 de octubre de 2012

\footnotetext{
Correspondencia a:

Armando Guevara agvillefort@yahoo.com
}

\section{Molecular characterization of carbapenem-resistant Pseudomonas aeruginosa from four hospitals of Venezuela}

We analyzed 17 strains of Pseudomonas aeruginosa resistant to carbapenems isolated in four hospitals in eastern and southern Venezuela collected between 2007 and 2010. Combined disk method showed production of metallo- $\beta$-lactamases (MBLs) in all strains. PCR and sequencing of genes encoding IMP, VIM and SPM metallo- $\beta$-lactamase families confirmed the presence of VIM-2 MBL in all strains. Pulsed field gel electrophoresis classified the strains into three similarity groups. The largest group, group A included 13 strains with over $83 \%$ similarity between them and was found in four hospitals. Two strains of Cumana hospital formed the group B and other two the Group C with similitary of $73 \%$ and $65 \%$ respectively with Group A. These results confirmed that hospitals in eastern and southern Venezuela, circulating strains of $P$. aeruginosa producing VIM-2 type MBLs with a common clonal origin. On the other hand, carbapenem-resistant $P$. aeruginosa circulating in Cumana city hospital had polyclonal origin.

Key words: Pseudomonas aeruginosa, metallo- $\beta$-lactamases, clonal dissemination.

Palabras clave: Pseudomonas aeruginosa, metalo- $\beta$-lactamasas, diseminación clonal.

\section{Introducción}

$P$ seudomonas aeruginosa, es un patógeno oportunista responsable de 10 a $15 \%$ de las infecciones asociadas a los cuidados de salud en todo el mundo ${ }^{1}$. La producción de metalo- $\beta$-lactamasas (MBLs) es uno de los principales mecanismos de resistencia con alta implicancia epidemiológica en $P$. aeruginosa debido a su capacidad de diseminación horizontal ${ }^{2}$. Estas enzimas se caracterizan por presentar un amplio perfil de sustratos, que incluye penicilinas, cefalosporinas y carbapenémicos, pero no hidrolizan a los monobactámicos (aztreonam) y su actividad es inhibida por el ácido etilendiaminotetraacético (EDTA) y otros quelantes de iones metálicos, limitando las opciones terapéuticas y favoreciendo el aumento de la morbi-mortalidad $^{2-4}$

Las MBLs pertenecen a la clase molecular B de Ambler y se subdividen en tres subclases: la subclase B1, que agrupa a las MBLs transferibles, inducibles o asociadas a plásmidos, y las subclases B2 y B3 que agrupan las MBLs cromosómicas derivadas de Aeromonas spp, Serratia fonticola y Stenotrophomonas maltophilia ${ }^{5}$. Dentro de las MBLs transferibles, se distinguen nueve grupos: IMP, VIM, SPM, GIM, AIM, NDM, SIM, DIM y KHM. Las primeras seis han sido encontradas en $P$. aeruginosa, siendo las IMP y VIM las más diseminadas en el mundo ${ }^{6,7}$. En Venezuela, las MBLs reportadas hasta ahora son de tipo VIM-2. El primer caso de infección por P. aeruginosa productor de MBL de tipo VIM-2 se publicó en el año 2004, en aislados de Caracas, en el marco del programa internacional de vigilancia SENTRY (Antimicrobial Surveillance Program $)^{8}$. Posteriormente, en el año 2009, se publicó el primer reporte donde se confirmó la presencia de cepas de $P$. aeruginosa productoras de MBL de tipo VIM-2 en el interior del país?.

Actualmente, en varios hospitales de Venezuela se siguen detectando cepas de $P$. aeruginosa con un perfil fenotípico compatible con la producción de MBLs ${ }^{10}$. Sin embargo, en pocos centros hospitalarios se ha hecho la confirmación molecular de este tipo de enzimas. Así mismo, se desconoce si existe una diseminación clonal de $P$. aeruginosa multi-resistente entre los diferentes servicios de un mismo centro hospitalario y, más grave aún, entre diferentes hospitales de una región o del país. Por tal motivo, en este estudio se analizaron 17 cepas de $P$. aeruginosa resistentes a los carbapenémicos provenientes de cuatro hospitales del oriente y sur de Venezuela colectados entre los años 2007 y 2010, con el fin de determinar la producción de MBLs y establecer su relación clonal.

\section{Material y Métodos}

\section{Cepas bacterianas}

Se analizaron 17 cepas de $P$. aeruginosa con resistencia a los carbapenémicos, aisladas de pacientes con infecciones asociadas a los cuidados de salud, hospitalizados en dife- 
rentes servicios del Hospital "Antonio Patricio de Alcalá" (HUAPA) de Cumaná, Hospital Industrial "Dr. Tulio Briceño M.” (HITB) de San Tomé, Complejo Hospitalario "Ruiz y Páez" (CHRP) de Ciudad Bolívar y Hospital "Raúl Leoni” (HRL) de San Félix, durante los años 2007 al 2010 (Figura 1). Las cepas procedentes de los distintos centros correspondieron a aislados derivados de manera espontánea por los centros para confirmación de mecanismos de resistencia al Hospital de Ciudad de Bolivar.

\section{Susceptibilidad antimicrobiana}

Fue determinada mediante el método de difusión con discos siguiendo los lineamientos descritos por el Clinical and Laboratory Standards Institute (CLSI) ${ }^{11}$. Se probaron los siguientes antimicrobianos: piperacilina $(100 \mu \mathrm{g})$, piperacilina/tazobactam $(100 / 10 \mu \mathrm{g})$, ceftazidima $(30 \mu \mathrm{g})$, cefoperazona $(75 \mu \mathrm{g})$, cefepima $(30 \mu \mathrm{g})$, imipenem $(10 \mu \mathrm{g})$, meropenem $(10 \mu \mathrm{g})$, aztreonam $(30 \mu \mathrm{g})$, amikacina $(30 \mu \mathrm{g})$, gentamicina $(10 \mu \mathrm{g})$, ciprofloxacina $(5 \mu \mathrm{g})$ y colistina $(10 \mu \mathrm{g})$. Como control de calidad se utilizó la cepa $P$. aeruginosa ATCC 27853.

\section{Detección fenotípica de las metalo-ß-lactamasas}

Se realizó mediante el método de discos combinados, utilizando discos de imipenem $(10 \mu \mathrm{g})$, meropenem $(10 \mu \mathrm{g})$ solos e impregnados con una solucion de ácido etilendiaminotetraacético/mercaptoacético de sodio (EDTA/SMA) $(0,5 \mathrm{M} / 300 \mathrm{ml})^{12}$. La solución de EDTA/ SMA se preparó de acuerdo a lo descrito por Lee et al ${ }^{13}$ y se conservó a $4^{\circ} \mathrm{C}$ hasta el momento de su utilización.

La detección de las MBLs se realizó inoculando placas con agar Mueller-Hinton (HiMedia ${ }^{\circledR}$ ), con suspensiones de las cepas ajustadas a una turbidez equivalente al patrón 0,5 de McFarland, luego se colocó dos discos de imipenem y dos discos de meropenem en cada placa, a un disco de cada carbapenémico se le agregó $10 \mu \mathrm{l}$ de la solución EDTA/SMA preparada previamente, las placas se incubaron a $35^{\circ} \mathrm{C}$ en aerobiosis durante $24 \mathrm{~h}$ y posteriormente se procedió a la lectura e interpretación de la prueba. Se consideró la prueba como positiva para la producción de MBLs, cuando el halo de inhibición de los carbapenémicos que contenían la solución de EDTA/ SMA fue al menos $5 \mathrm{~mm}$ mayor con respecto al halo de los carbapenémicos sin dicha solución ${ }^{12}$. Como control de calidad de la solución EDTA/SMA y de las pruebas de detección fenotípica de las metalo-enzimas, se utilizó la cepa de $P$. aeruginosa ATCC 27853 como control negativo y $P$. aeruginosa 77297 productora de MBL de tipo VIM-2 como control positivo.

\section{Detección de genes que codifican para las metalo- $\beta$-lactamasas mediante reacción de polimerasa en cadena (RPC)}

Extracción del ADN cromosomal de las cepas de P. aeruginosa. A partir de un cultivo puro de $18 \mathrm{~h}$ en agar tripticasa soya (Hi Media $\left.{ }^{\circledR}\right)$ se tomaron varias colonias, se resuspendieron en $200 \mu \mathrm{l}$ de TE ( $10 \mathrm{mM}$ Tris, $1 \mathrm{mM}$ EDTA) y se sometieron a ebullición en baño de María por 10 min. Posteriormente, por centrifugación, se separó el sobrenadante conteniendo el ADN bacteriano, el cual se transfirió a tubos para microcentrífuga y se almacenó a $-20^{\circ} \mathrm{C}^{9}$.

La presencia de los genes que codifican para las MBLs de las familias SPM, VIM e IMP se realizó a través de la RPC utilizando los iniciadores mostrados en la Tabla 1. Las amplificaciones se llevaron a cabo en un volumen final de $50 \mu \mathrm{l}$ y la mezcla estuvo compuesta por $1 \mu \mathrm{lde}$ cada iniciador ( $5 \mathrm{pmol} / \mathrm{ml}) ; 20,5 \mathrm{ml}$ agua bidestilada ultrapura; $25 \mu \mathrm{l} \mathrm{LPU}$ (Fundaim ${ }^{\circledR}$ ) y $2,5 \mu \mathrm{l}$ de ADN. Las condiciones de amplificación fueron la descritas anteriormente ${ }^{3,14,15}$. Los productos obtenidos de las amplificaciones por RPC fueron observados en corridas electroforéticas en geles

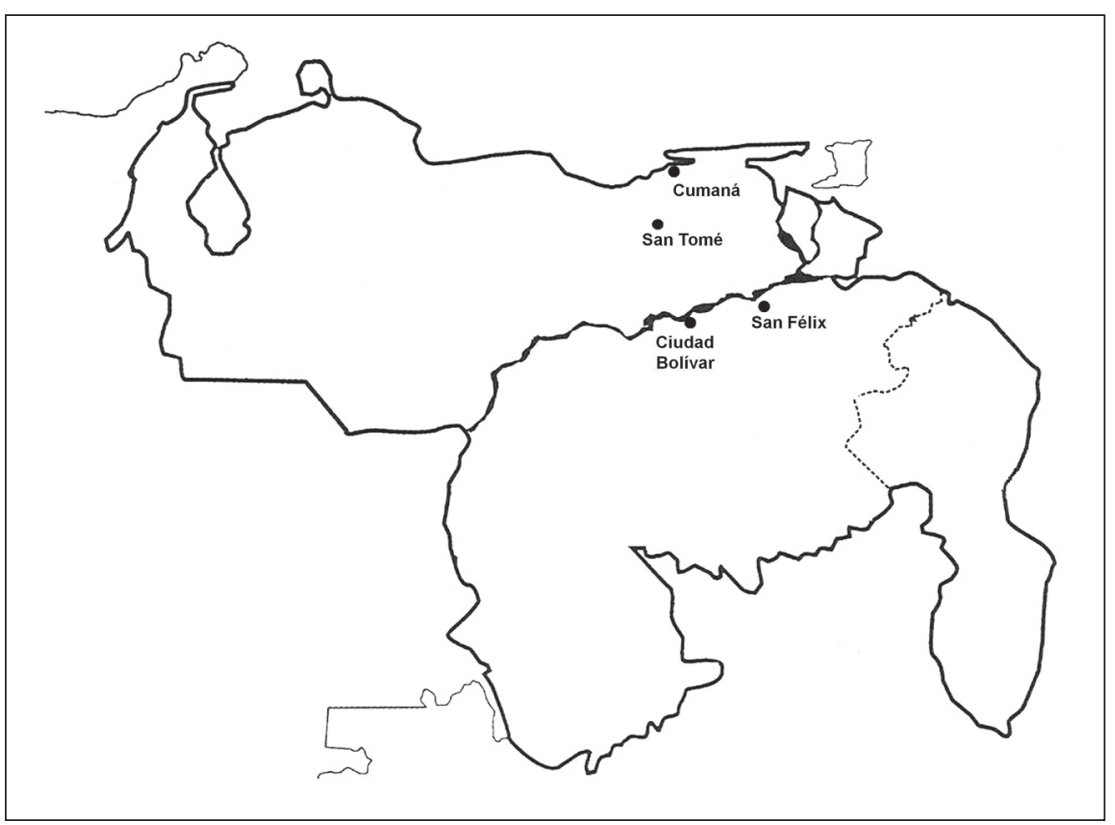

Figura 1. Ubicación geográfica de los cuatro hospitales participantes en el estudio.

\begin{tabular}{|c|c|c|c|c|}
\hline Iniciador & Secuencia & $\begin{array}{c}\text { Gen } \\
\text { amplificado }\end{array}$ & $\begin{array}{c}\text { Tamaño del } \\
\text { producto } \\
\text { esperado }\end{array}$ & Referencia \\
\hline $\begin{array}{l}\text { SPM F } \\
\text { SPM R }\end{array}$ & $\begin{array}{l}\text { 5'-CCTACAATCAACGGCGA CC-3' } \\
5^{\prime} \text {-TCGCCGTGTCCAGGTATAAC-3' }\end{array}$ & $b / a_{\mathrm{SPM}}$ & $786 \mathrm{pb}$ & 14 \\
\hline $\begin{array}{l}\text { IMPF } \\
\text { IMPR }\end{array}$ & $\begin{array}{l}\text { 5'-CTACCGCAGCAGAGTCTTTG-3' } \\
\text { 5'-AACCAGTTTTGCCTTACCAT-3' }\end{array}$ & $b / a_{\mathbb{I M P}}$ & $587 \mathrm{pb}$ & 15 \\
\hline $\begin{array}{l}\text { VIM 2004A } \\
\text { VIM 2004B }\end{array}$ & $\begin{array}{l}\text { 5'-GTTTGGTCGCATATCGCAAC-3' } \\
5^{\prime} \text {-AATGCGCAGCACCAGGATAG-3' }\end{array}$ & $b / a_{\text {VII }}$ & $382 \mathrm{pb}$ & 3 \\
\hline
\end{tabular}


de agarosa al 1,5\% (Promega $\left.{ }^{\circledR}\right)$, teñidos con bromuro de etidio $\left(\right.$ Sigma $\left.{ }^{\circledR}\right)$ y fotografiados con una cámara digital PowerShot A700 (Canon $\left.{ }^{\circledR}\right)$. Como marcador del tamaño molecular se utilizó Ladder 50 pb (Promega $\left.{ }^{\circledR}\right)$.

Secuenciación de los productos amplificados. Los productos de la RPC que resultaron positivos para la detección de genes que codifican para las MBLs se purificaron utilizando el kit Wizard DNA Clean-Up System (Promega $\left.{ }^{\circledR}\right)$. Posteriormente, las secuencias nucleotídicas fueron determinadas por el Centro de Secuenciación y Análisis de Ácidos Nucleicos (CeSAAN) del Instituto Venezolano de Investigaciones Científicas (IVIC), Caracas-Venezuela, mediante electroforesis capilar en un secuenciador de ácidos nucleicos modelo ABI 3130XL (Applied Biosystems $\left.{ }^{\circledR}\right)$, utilizando los mismos iniciadores utilizados en la RPC. Las secuencias nucleotídicas resultantes fueron analizadas mediante el uso del programa Basic Local Alignment Search Tool (BLAST) del National Center for Biotechnology Information (NCBI) y comparadas con las secuencias génicas incluidas en la base de datos (http:// www.ncbi.nlm.nih.gov/blast/).

Tipificación molecular de los aislados de P. aeruginosa. Se realizó la tipificación molecular de los aislados mediante la electroforesis de campos pulsantes (ECP) en el equipo Guefast-06® (NEURONIC, S.A) según lo publicado anteriormente ${ }^{16}$ con algunas modificaciones.

Preparación del ADN inmovilizado. Las cepas de $P$. aeruginosa fueron crecidas en agar Mueller-Hinton (HiMedia ${ }^{\circledR}$ ), luego fueron recolectadas y lavadas con una solución de $\mathrm{NaCl}$ al 0,5\% pH 8,0; posteriormente, se suspendieron en $50 \mu \mathrm{l}$ de la solución de lavado y se mezclaron con $150 \mu \mathrm{l}$ de agarosa de bajo punto de fusión al 1,5\%, fundida y atemperada a $45{ }^{\circ} \mathrm{C}$ y utilizando el molde para la fabricación de bloques, se prepararon minibloques de $3 \mathrm{~mm}$ x $3 \mathrm{~mm}$ x 0,7 mm (largo, ancho y grosor). La concentración final de células bacterianas en cada minibloque fue de $2,1 \times 10^{9}$ céls $/ \mathrm{ml}$ aproximadamente.

Una vez elaborados los minibloques, las células fueron sometidas a un proceso de lisis y desproteinización colocándolas en solución que contenía $0,01 \mathrm{M}$ Tris; $0,1 \mathrm{M}$ EDTA; $1 \%$ sarcosyl; $1 \%$ nonident $\mathrm{P} 40$ y $4 \mathrm{M}$ urea $\mathrm{pH}$ 9,5 y se incubaron por $18 \mathrm{~h}$ a $43{ }^{\circ} \mathrm{C}$, posteriormente se eliminó esta solución y se lavaron con agua destilada y con una solución $0,01 \mathrm{M}$ Tris-HCL y $0,1 \mathrm{M}$ EDTA $\mathrm{pH}$ 8 , donde se conservaron a $4^{\circ} \mathrm{C}$ hasta el momento de la digestión.

Digestión del ADN inmovilizado con enzimas de restricción. Cada minibloque fue lavado tres veces en solución 0,01M Tris-HCL y 0,05 M EDTA pH 8; a $4^{\circ} \mathrm{C}$ por $10 \mathrm{~min}$, luego se incubaron con $100 \mu \mathrm{l}$ de tampón de digestión D 1X de la enzima proporcionado por el fabricante (Promega ${ }^{\circledR}$ ) a temperatura ambiente por 10 min. Posteriormente, cada minibloque fue transferido a $100 \mu \mathrm{l}$ de tampón de digestión fresco y se colocó $1 \mu \mathrm{l}$ de la enzima de restricción $\mathrm{XbaI}$ (Promega $\left.{ }^{\circledR}\right)$, se incubó durante $4 \mathrm{~h}$ a $37^{\circ} \mathrm{C}$, la reacción se detuvo reemplazando el tampón por $1 \mathrm{ml}$ de $0,01 \mathrm{M}$ Tris-HCL: 0,1M EDTA pH 8,0.

Electroforesis de campo pulsado de los fragmentos de macrorestricción del ADN de P. aeruginosa. La electroforesis se realizó en una cámara minichef del equipo Guefast-06® (NEURONIC, S. A), en un gel de $7 \mathrm{~cm}$ x 5 $\mathrm{cm}$ x 0,5 cm (largo, ancho, grosor); se utilizaron $400 \mathrm{ml} \mathrm{de}$ tampón Tris-Borato EDTA $0,5 \mathrm{X}$ a $20^{\circ} \mathrm{C}$. Los fragmentos de ADN fueron separados a $10 \mathrm{~V} / \mathrm{cm}$ durante $4 \mathrm{~h}, 57$ min y $30 \mathrm{~s}$ en un gel de agarosa al $1,5 \%$ sumergido en el tampón de electroforesis. Los fragmentos de restricción fueron resueltos programando las siguientes rampas con diferentes números de pulsos y tiempos de pulso: 5 pulsos por $20 \mathrm{~s}, 15$ pulsos por $15 \mathrm{~s}, 320$ pulsos por $10 \mathrm{~s}, 1.020$ pulsos por $5 \mathrm{~s}$ y 100 pulsos por $3 \mathrm{~s}$. El gel se coloreó durante $30 \mathrm{~min}$ en una solución $0,5 \mu \mathrm{g} / \mathrm{ml}$ de bromuro de etidio (Sigma-Aldrich ${ }^{\circledR}$ ), luego se visualizó en un transiluminador UV y se fotografió con una cámara digital PowerShot A700 (Canon $®)$. El tamaño de los fragmentos de restricción en kilopares de bases $(\mathrm{Kb})$, se calculó al comparar la posición de las bandas con la co-migración de un marcador de peso molecular como el fago lambda concatamerizado de 48,5 Kb (Sigma-Aldrich ${ }^{\circledR}$ ).

El análisis de los perfiles obtenidos por ECP y la construcción del dendograma fueron realizados mediante el análisis computarizado, utilizando el software GuefaScan ${ }^{\circledR}$ (NEURONIC S.A.), acorde con el coeficiente de similaridad de Dice y el algoritmo UPGMA (Unweighted Pair Group Method with Arithmetic Averages). Las cepas que presentaron índice de similaridad igual o mayor a $80 \%$ se consideraron clonalmente relacionadas ${ }^{17}$.

\section{Resultados}

Se evaluaron 17 cepas de $P$. aeruginosa de origen clínico con resistencia a los carbapenémicos, procedentes de cuatro hospitales del oriente y sur de Venezuela, cuyo perfil de susceptibilidad demostró siete patrones diferentes designados por orden alfabético de la A hasta la G. El patrón más frecuente fue el A (8/17), caracterizado por resistencia a los $\beta$-lactámicos, aminoglucósidos y fluoroquinolonas, sensibilidad intermedia a aztreonam y sensibilidad a colistina. Seguido del patrón B (4/17), el cual se diferenció del A por demostrar sensibilidad a aztreonam. El patrón A se observó en dos cepas del CHRP, dos del HITB y cuatro del HUAPA, mientras que el patrón 
B se presentó en una cepa de cada hospital en estudio. Los patrones C, D, E, F y G fueron los menos frecuentes, observándose cada uno en una cepa. Independientemente del patrón de susceptibilidad que presentaron las cepas de $P$. aeruginosa, todas fueron fenotípicamente productoras de MBLs por el método de discos combinados y a través de la RPC y posterior secuenciación, se detectó MBLs de tipo VIM-2 en todas las cepas (Tabla 2).

El análisis de los productos de restricción obtenidos al digerir el genoma de las cepas de $P$. aeruginosa productoras de MBL de tipo VIM-2 con la enzima XbaI, permitió identificar tres grupos de similaridad designados como A, B y C. El grupo dominante (A) estuvo constituido por 13 cepas con similaridad superior a $83 \%$, a su vez este grupo se subdividió en cuatro subgrupos: El A1, subgrupo dominante, estuvo conformado por dos cepas del CHRP, dos del HUAPA y tres del HITB, indistinguibles entre sí; el subgrupo A2, compuesto por todas las cepas del HRL, presentando $97 \%$ de similaridad con el subgrupo A1; A3 conformado por dos cepas del CHRP con $86 \%$ de similaridad con A1 y el subgrupo A4 con una cepa del CHRP que presentó $83 \%$ de similaridad con A1. Dos cepas del HUAPA conformaron el Grupo B y otras dos cepas de este mismo centro, el Grupo C con similaridad de 73 y $65 \%$, respectivamente, en relación al Grupo A.

Al comparar los patrones de similaridad que se presentaron en cada hospital, se observó que todas las cepas de $P$. aeruginosa del CHRP (5/5) pertenecían a un mismo grupo de similaridad (A). Sin embargo, se encontró que dependiendo del servicio hospitalario de donde provenía el aislado, se ubicaron en diferentes subgrupos; las cepas de UCI y del servicio de medicina pertenecen al subgrupo A1, mientras que las cepas de cirugía (2) y pediatría (1) se ubicaron en los subgrupos A3 y A4 respectivamente. Las cepas de $P$. aeruginosa del HRL (3/3), presentaron el mismo patrón de bandas, por tanto, pertenecían al mismo subgrupo de similaridad (A2) independientemente del servicio evaluado. En el HITB, las cepas de $P$. aeruginosa (3/3) fueron indistinguibles entre sí formando parte del subgrupo A1; sin embargo, todas las cepas provenían de la UCI, por lo que no se pudo evaluar si existía una relación entre las cepas de $P$. aeruginosa provenientes de la UCI con respecto a otros servicios.

Las cepas de $P$. aeruginosa del HUAPA fueron las que presentaron mayor variabilidad en los patrones de bandas obtenidos en la ECP, distribuyéndose en los tres grupos de similaridad encontrados. Dos cepas, una de la UCI de adultos y otra del servicio de medicina se ubicaron en el grupo de similaridad A (subgrupo A1). Una cepa de la UCI pediátrica y otra de la UCI de adultos conformaron el grupo B, mientras que el último grupo de similaridad (C) estuvo constituido por una cepa de retén y otra del servicio de medicina (Figura 2).

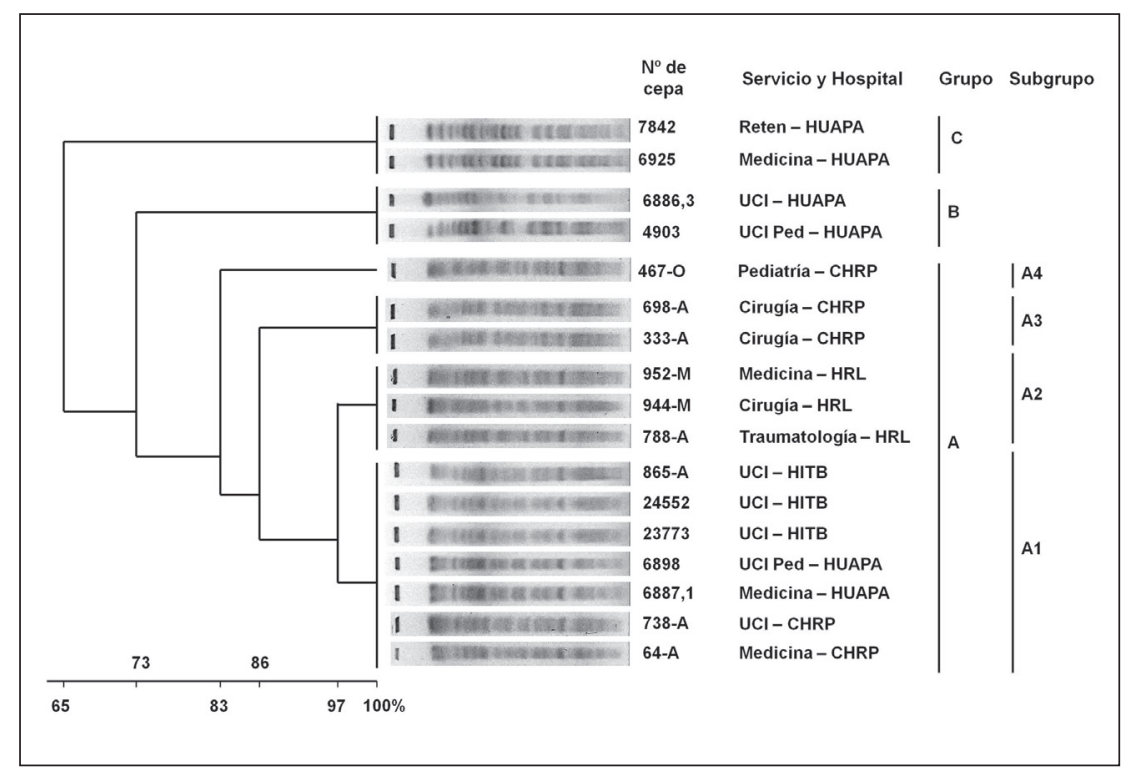

Figura 2. Dendograma de las cepas de $P$. aeruginosa con base en sus patrones de la electroforesis de campos pulsantes. HUAPA: Hospital Universitario "Antonio Patricio Alcalá", CHPR: Hospital Universitario "Ruiz y Páez", HRL. Hospital "Raúl Leoni", HITB: Hospital Industrial "Tulio Briceño". UCl; Unidad de Cuidados Intensivos, UCI Ped: Unidad de Cuidados Intensivos Pedíatricos.

\section{Discusión}

Las cepas de $P$. aeruginosa productoras de MBLs frecuentemente están relacionadas a las infecciones asociadas a los cuidados de salud, y generalmente presentan otros mecanismos de resistencia, que reducen aún más las opciones terapéuticas disponibles para tratar dichas infecciones. En este estudio se evaluaron 17 cepas de $P$. aeruginosa, provenientes de diferentes servicios de cuatro hospitales de Venezuela con el fin de describir el comportamiento de uno de los mecanismos de resistencia más importantes que se presenta en nuestros hospitales.

Las pruebas de susceptibilidad demostraron que todas las cepas de $P$. aeruginosa presentaron un perfil de susceptibilidad compatible con la producción de MBLs, y que en la mayoría (16/17), la resistencia a los $\beta$-lactámicos de amplio espectro estuvo asociada a la resistencia a gentamicina, amikacina y ciprofloxacina, lo cual podría deberse a la presencia de otros mecanismos de resistencia como: las enzimas modificadoras de aminoglucósidos, bombas de expulsión, alteraciones de la permeabilidad de la pared celular, entre otros, ya que $P$. aeruginosa presenta una marcada capacidad para adquirir y expresar diferentes mecanismos de resistencia simultáneamente ${ }^{2,7,18}$.

Aztreonam no se encuentra dentro del perfil de hidrólisis de las MBLs, por lo cual representa una opción terapéutica para el tratamiento de las infecciones producidas por $P$. aeruginosa productoras de este tipo de enzimas. Sin embargo, en este estudio, sólo 5/17 cepas fueron 


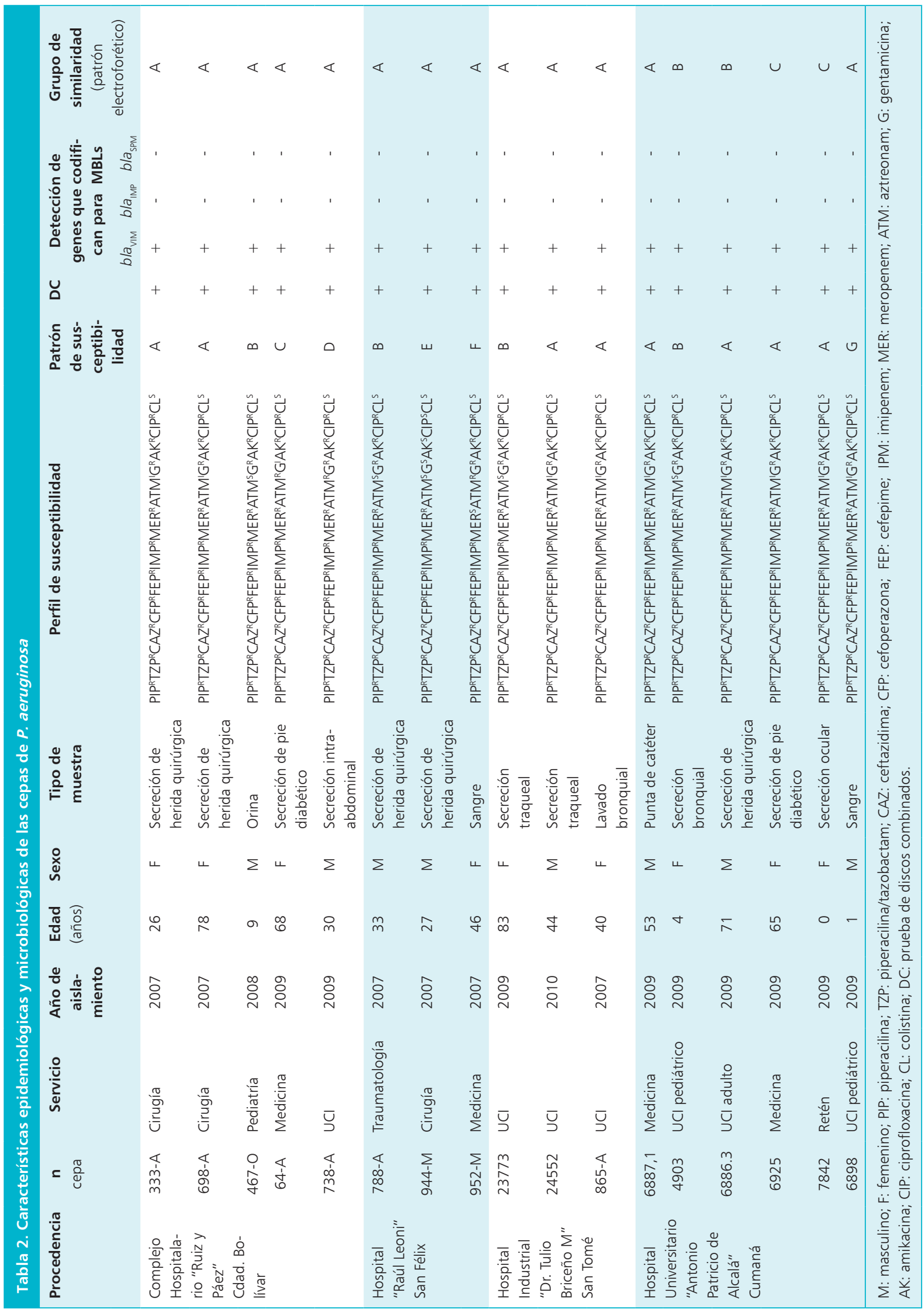


sensibles al aztreonam, lo cual coincide con estudios realizados en los últimos años en diferentes regiones de Venezuela donde se han venido reportando, cada vez con mayor frecuencia, la presencia de cepas de $P$. aeruginosa productoras de MBLs con resistencia al aztreonam. Esta resistencia generalmente se debe a la producción de $\square$ lactamasas de espectro extendido (BLEE), las cuales son enzimas que tienen la capacidad de hidrolizar y causar resistencia a los $\beta$-lactámicos incluyendo aztreonam ${ }^{10,19}$.

Al hacer un análisis del comportamiento de la susceptibilidad a aztreonam en los hospitales estudiados, se encontró que, de las cinco cepas procedentes del CHRP, dos presentaron susceptibilidad intermedia a aztreonam y dos fueron resistentes, lo cual difiere al estudio realizado en el año 2009, donde las diez cepas de $P$. aeruginosa evaluadas procedentes de la UCI de dicho hospital presentaron susceptibilidad a este fármaco ${ }^{9}$, lo cual podría indicar que con el transcurso del tiempo las cepas de $P$. aeruginosa del CHRP han adquirido nuevos mecanismos de resistencia, quizás por el uso indiscriminado de los antimicrobianos. En el HRL una cepa fue resistente a aztreonam mientras que en el HITB y el HUAPA se observaron cepas con susceptibilidad intermedia a este antimicrobiano. En estos hospitales no hay estudios anteriores que permitan evaluar cómo ha evolucionado la resistencia a este antimicrobiano.

Estudios previos refieren que colistina presenta una potente actividad frente a cepas de $P$. aeruginosa multiresistentes, por lo que ha surgido como una alternativa terapéutica para las infecciones causadas por este microorganismo ${ }^{18,20-22}$. Sin embargo, se ha reportado resistencia a colistina en algunos aislados ${ }^{23-25}$. En este estudio, todas las cepas de $P$. aeruginosa (17/17) fueron susceptibles a colistina, lo cual coincide con evaluaciones previas realizadas en cepas nosocomiales y de la comunidad en Mérida-Venezuela ${ }^{18}$. Sin embargo, se debe mantener una vigilancia constante a dicho antimicrobiano, ya que es una de las pocas alternativas terapéuticas para el tratamiento de las infecciones causadas por $P$. aeruginosa y otros bacilos gramnegativos multi-resistentes.

En Venezuela, la primera detección de MBL de tipo VIM-2 fue en el año 2004 en tres cepas de $P$. aeruginosa de origen clínico provenientes de un hospital de Caracas ${ }^{8}$. Posteriormente, se reportaron cepas de $P$. aeruginosa productoras de MBL de la familia VIM en hospitales de Caracas y Ciudad Bolívar ${ }^{10}$ y luego se confirmó la presencia de MBL de tipo VIM-2 en cepas de $P$. aeruginosa en el sur del país ${ }^{9}$, lo cual coincide con nuestro estudio donde todas las cepas de $P$. aeruginosa fueron productoras de MBL de tipo VIM-2.

Al evaluar los resultados de la relación clonal mediante ECP se confirmó que en los hospitales de Cumaná, San Tomé, Ciudad Bolívar y San Félix, circulan cepas de $P$. aeruginosa productoras de MBL de tipo VIM-2, con un origen clonal común, lo cual es atribuible a la transferencia entre hospitales de pacientes colonizados o infectados, debido a la cercanía que existe entre ellos. Resultados similares han sido reportados en estudios previos en hospitales de Japón, Taiwan, Hungría y Brasil donde se ha evaluado y comprobado la diseminación clonal de cepas de $P$. aeruginosa productoras de MBLs entre hospitales de una o de diferentes regiones ${ }^{26-30}$. La presencia de la diseminación clonal de cepas de $P$. aeruginosa productoras de MBL de tipo VIM-2 en hospitales de diferentes regiones de Venezuela indica prácticas inadecuadas de control de las infecciones asociadas a los cuidados de salud, posiblemente por la falta de adherencia a los protocolos de control de infecciones.

En este estudio, además de las cepas de origen clonal común, se observó la presencia de tres clones diferentes en uno de los hospitales, lo que podría indicar no sólo la diseminación de una cepa resistente, sino la diseminación horizontal de este mecanismo de resistencia, lo cual puede deberse a la difusión de plásmidos de resistencia producto de la presión selectiva ejercida por el abuso y mal uso de los antimicrobianos, tal como ha sido reportado por otros autores ${ }^{31}$.

En el CHRP, se observó que las cepas de P. aeruginosa productoras de MBL de tipo VIM-2 pertenecían a un mismo grupo clonal con subgrupos de similaridad diferentes, dependiendo del servicio hospitalario donde fueron aisladas, lo que hace presumir que todas estas cepas provienen de un ancestro común, que posiblemente adquirió un determinante de resistencia y posteriormente se diseminó por los diferentes servicios. Estos hallazgos son similares a los reportados en Colombia, donde se encontró que algunas cepas de $P$. aeruginosa productoras de MBLs con diferentes perfiles de resistencia, pertenecían a un mismo grupo clonal ${ }^{32}$.

En el HRL se encontró que todas las cepas pertenecían al mismo subgrupo de similaridad independientemente del servicio de aislamiento, lo cual indica una clara diseminación clonal de $P$. aeruginosa productora de MBL de tipo VIM-2 en los servicios de dicho hospital. Resultados similares han sido reportados en España ${ }^{4}$. En el HITB todas las cepas fueron indistinguibles entre si y conformaron un mismo subgrupo de similaridad, pero, a diferencia del HRL, pertenecían sólo al servicio de la UCI por lo que se sugiere realizar investigaciones posteriores donde se evalué si existe una diseminación clonal de estas cepas en los diferentes servicios del hospital de San Tomé.

En el HUAPA se evidenció la presencia de cepas de $P$. aeruginosa productoras de MBL de tipo VIM-2 de origen policlonal. En diferentes servicios se encontró cepas de $P$. aeruginosa de los grupos clonales A, B y C. Coincidiendo con estudios realizados en Argentina y España donde demuestran la diseminación de varios grupos clonales de cepas de $P$. aeruginosa productoras de MBLs en un mismo 
hospital $^{2,22}$. Es importante destacar que el grupo clonal B se observó en cepas provenientes de la UCI de adultos y de la UCI pediátrica, mientras que el clon $\mathrm{C}$ provenía de cepas de los servicios de reten y medicina y que además estos grupos clonales presentan una similaridad de $92 \%$ entre ellos, lo cual podría indicar que conforman un solo grupo clonal, debido a la similitud que existe entre ellos y la relación que existe entre los servicios de donde proceden los aislados.

Finalmente, este estudio permitió evidenciar que existe una diseminación clonal de $P$. aeruginosa productoras de MBL de tipo VIM-2 en los hospitales del oriente y sur de Venezuela, implicando un impacto significativo en las opciones terapéuticas para las infecciones causadas por este tipo de microrganismo. Por ello, en los laboratorios de microbiología se deben implementar métodos microbiológicos que provean información sobre los mecanismos de resistencia que permitan garantizar el control de las infecciones causadas por esta bacteria y la introducción oportuna de las medidas de prevención. Así mismo, se deben implementar programas de vigilancia epidemiológica que permitan racionalizar el uso de antimicrobianos de amplio espectro basados en la epidemiología local y el mecanismo de resistencia presente, así como aplicar técnicas de biología molecular para la identificación de genes de resistencia y la tipificación de microorganismos, para reducir el surgimiento y difusión de mecanismos de resistencia.
Agradecimientos. A Elsa Salazar, Benito Campos, Yasmin Rodríguez y Margarita Echeverría por facilitarnos las cepas usadas en este estudio.

\section{Resumen}

Se analizaron 17 cepas de Pseudomonas aeruginosa resistentes a carbapenémicos aisladas en cuatro hospitales del oriente y sur de Venezuela colectadas entre los años 2007 y 2010. En todas las cepas se demostró la producción de metalo- $\beta$-lactamasas (MBLs) por el método de discos combinados. Mediante la amplificación por la reacción de polimerasa en cadena (RPC) de los genes que codifican para las metalo- $\beta$-lactamasas de las familias IMP, VIM y SPM, y su posterior secuenciación, se confirmó la presencia de MBLs de tipo VIM-2 en todas las cepas. Mediante la electroforesis de campos pulsantes (ECP), las cepas se clasificaron en tres grupos de similaridad. El grupo dominante (A) estuvo constituido por 13 cepas provenientes de los cuatro hospitales, con similaridad superior al $83 \%$ entre ellas. Dos cepas del hospital de Cumaná conformaron el Grupo B y otras dos el Grupo C con similaridad de 73 y $65 \%$, respectivamente, con el Grupo A. Estos resultados confirmaron que en los hospitales del oriente y sur de Venezuela, circulan cepas de $P$. aeruginosa productoras de MBLs de tipo VIM-2, con un origen clonal común. Asimismo, en el hospital de Cumaná circulan cepas de origen policlonal.

\section{Referencias bibliográficas}

1.- Driscoll J, Brody S, Kollet M. The epidemiology, pathogenesis and treatment of Pseudomonas aeruginosa infections. Drugs 2007; 67: 351-68.

2.- Cejas D, Almuzara M, Santella G, Tuduri A, Palombarani S, Figueroa S, et al. Caracterización fenotípica y genotípica de la resistencia a imipenem en Pseudomonas aeruginosa aisladas en un hospital de Buenos Aires. Rev Arg Microbiol 2008; 40: 238-45.

3.- Pitout J, Chow B, Gregson D, Laupland K, Elsayed S, Church D. Molecular epidemiology of metallo- $\beta$-lactamase producing Pseudomonas aeruginosa in the Calgary Health Region: Emergence of VIM-2 producing isolates. J Clin Microbiol 2007; 45: 294-8.

4.- Rodríguez M, Ruiz B, Rodríguez C, Romo M, Monteagudo I y Martínez L. Caracterización molecular de aislados clínicos de Pseudomonas aeruginosa productores de la metalo- $\beta$ lactamasa VIM-2 en Cantabria, España. Enferm Infecc Microbiol Clin 2010; 28: 99-103.

5.- Walsh T, Toleman M, Poirel L, Nordmann P. Metallo- $\beta$-lactamasas: the quiet before the storm?. Clin Microbiol Rev 2005; 18: 306-25.
6.- Jovcic B, Lepsanovic Z, Suljagic V, Rackov G, Begovic J, Topisirovic L, et al. Emergence of NDM-1 metallo- $\beta$-lactamase in Pseudomonas aeruginosa clinical isolates from Serbia. Antimicrob Agents Chemother 2011; 55: 3929-31.

7.- Nicolau J, Oliver A. Carbapenemasas en especies del género Pseudomonas. Enferm Infecc Microbiol Clin 2010; 28: 19-28.

8.- Mendes R, Costanheira M, García P, Guzmán M, Toleman M, Walsh T, et al. First isolation of blaVIM-2 in Latin America: report from the SENTRY antimicrobial surveillance program. Antimicrob Agents Chemother 2004; 48: 1433-4.

9.- Guevara A, Waard J, Araque M. Detección del gen blaVIM-2 de Pseudomonas aeruginosa productoras de metalo- $\beta$-lactamasa aisladas en una unidad de cuidados intensivos en Ciudad Bolívar, Venezuela. Rev Chilena Infectol 2009; 26: 336-41.

10.- Sánchez D, Marcano D, Spadola E, León L, Payares D, Ugarte C, et al. Metaloenzimas tipo VIM detectadas en aislamientos clínicos en Pseudomonas aeruginosa en cuatro hospitales en Venezuela. Rev Inst Nac Hig "Rafael Rangel" 2008; 39: 17-22.
11.- Clinical and Laboratory Standards Institute. Performance standards for antimicrobial susceptibility testing; tenth edition informational supplement. M02-A10. Pensilvania, USA: 2010.

12.- Guevara A, Gamboa A, Machado M, Vera M. Evaluación del ácido etilendiaminotetraacético y del mercaptoacético de sodio en la detección de metalo- $\beta$-lactamasas en Pseudomonas aeruginosa mediante la técnica del disco combinado. Rev Soc Venez Microbiol 2010; 30: 11-7.

13.- Lee K, Lim Y S, Yong D, Yum J H, Chong Y. Evaluation of the Hodge test and the imipenem-EDTA double-disk synergy test for differentiating metallo- $\beta$-lactamase-producing isolates of Pseudomonas spp and Acinetobacter spp. J Clin Microbiol 2003; 41: 4623-9.

14.- Gales A, Menezes L, Silbert S, Sader H. Dissemination in distinct Brazilian regions of an epidemic carbapenem-resistant Pseudomonas aeruginosa producing SPM metallo- $\beta$-lactamase. J Antimicrob Chemother 200; 52: 699-702.

15.- Senda K, Arakawa Y, Ichiyama S, Nakashima K, Ito $\mathrm{H}$, Ohsuka S, et al. RCP detection of metalo$\beta$-lactamase gene $\left(\right.$ bla $\left._{\mathrm{IMP}}\right)$ in gram-negative rods resistant to broad-spectrum $\beta$-lactams. J Clin Microbiol 1996; 34: 2909-13. 
16.- López-Cánovas L, Sánchez-Alonso A, Higginson D, Ariosa C, Clark H, Riverón A M. Nonenzymatic protocol for Pseudomonas aeruginosa DNA preparation and rapid subtyping by mini pulsed-field gel electrophoresis. Electrophoresis 2003; 24 1152-8.

17.- Van Belkum A, Tassios P, Dijkshoorn L, Haeggman S, Cookson B, Fry N, et al. Guidelines for the validation and application of typing methods for use in bacterial epidemiology. Clin Microbiol Infect 2007; 13: 1-46.

18.- López F, Culebras E, Bonilla I, Gómez M, Picazo J. Actividad in vitro de terapia combinada con colistina frente a Pseudomonas aeruginosa aisladas en unidad de cuidados intensivos. Rev Esp Quimioter 2008; 21: 189-93.

19.- Salazar P, Araque M, Mosqueda N. Análisis fenotípico del gen bla ${ }_{\mathrm{VIM}}$ en cepas de Pseudomonas aeruginosa productoras de metalo- $\beta$-lactamasas aisladas en Mérida, Venezuela. Rev Fac Farm 2010; 52: 12-7.

20.- Salinas C, Hernández A, Oropeza R, Olvera C, Poblano M, Franco J. Colistín en el tratamiento de infección por Pseudomonas aeruginosa multidrogorresistente. Rev Asoc Mex Med Crit y Ter Int 2010; 24: 173-7.

21.- Durakovic N, Radojcic V, Boban A, Mrsic M, Sertic D, Serventi R, et al. Efficacy and safety of colistin in the treatment of infections caused by multidrug-resistant Pseudomonas aeruginosa in patients with hematologic malignancy: a matched pair analysis. Intern Med 2011; 50: 1009-13.

22.- Jimeno A, Alcalde M, Blázquez A. Detección de un brote epidémico por Pseudomonas aeruginosa multirresistente productora de metalo-beta-lactamasa. Rev Clin Esp 2011; 1-5.

23.- Laupland K, Parkins M, Church D, Gregson D, Louie T, Conly J, et al. Population-based epidemiological study of infections caused by carbapenem-resistant Pseudomonas aeruginosa in the Calgary health region: Importance of metallo- $\beta$-lactamase $(\mathrm{M} \beta \mathrm{L})$ producing strains. $\mathrm{J}$ Infect Dis 2005; 192: 1606-12.

24.- Fica A, Céspedes I, Gompertz M, Jalón M, Sakurada A, Sáez E. Colistín en infecciones nosocomiales por bacilos gramnegativos panresistentes. Rev Chilena Infectol 2007; 24 : 360-7.

25.- Samonis G, Matthaiou D, Kofteridis D, Maraki S, Falagas M. In vitro susceptibility to various antibiotics of colistin-resistant gramnegative bacterial isolates in a general tertiary hospital in Crete, Greece. Clin Infect Dis 2010; 50: 1689-91.

26.- Libisch B, Muzslay M, Gacs M, Minárovits J, Knausz M, Watine J, et al. Molecular epidemiology of VIM-4 metallo- $\beta$-lactamase producing Pseudomonas sp. isolates in Hungary. Antimicrob Agents Chemother 2006; 50:
4220-3.

27.- Huang Y, Changa S, Lauderdaleb T, Yanga A, Wang J. Molecular epidemiology of carbapenem-resistant Pseudomonas aeruginosa carrying metallo- $\beta$-lactamase genes in Taiwan. Diag Microbiol Infec Dis 2007; 59: 211-6.

28.- Ohara M, Kouda S, Onodera M, Fujiue Y, Sasaki M, Kohara T, et al. Molecular characterization of imipenem-resistant Pseudomonas aeruginosa in Hiroshima, Japan. Microbiol Inmunol 2007; 51: 271- 7.

29.- Scheffer M, Gales A, Barth A, Rodrigues J, Dalla L. Carbapenem-resistant Pseudomonas aeruginosa. Clonal spread in southern Brazil and in the state of Goiás. Braz J Infect Dis 2010; 14: 508-9.

30.- Souza A, Rodrigues M, Dorn G, Souza M, Pereira N, Garcia B, et al. Metallo- $\beta$-lactamase and genetic diversity of Pseudomonas aeruginosa in intensive care units in Campo Grande, MS, Brazil. Braz J Infect Dis 2011; 15: 195-9.

31.- Peleg A, Franklin C, Bell J, Spelman D. Dissemination of the metallo- $\beta$-lactamase gene blaIMP-4 among Gram-negative pathogens in a clinical setting in Australia. Clín Infect Dis 2005; 41: 1549-56.

32.- Martínez P, Espinal P, Máttar S. Epidemiología molecular de Pseudomonas aeruginosa resistente a $\beta$-lactámicos de amplio espectro en el hospital San Jerónimo de Montería. Infectio 2007; 11: 6-15. 